\title{
Exercise mode and attentional networks in older adults: A cross-sectional study
}

\author{
Biye Wang $^{1,2}$, Wei Guo ${ }^{\text {Corresp. 1,2 }}$ \\ ${ }^{1}$ College of Physical Education, Yangzhou University, Yangzhou, Jiangsu, China \\ 2 Institute of Sports, Exercise and Brain, Yangzhou University, Yangzhou, Jiangsu, China \\ Corresponding Author: Wei Guo \\ Email address: guowei_sus@outlook.com
}

Background Previous studies have indicated that physical exercise enhances attentional function; however, the relationship between exercise mode and attentional networks has not been clarified for older adults ( $>60$ years old). This study aimed to investigate the relationship between attentional networks and different exercise modes in older adults.

Methods Two hundred and fifty-nine participants aged between 60 to 81 years were enrolled and classified into three groups (closed-skill group, open-skill group, or sedentary control group) using an exercise-related questionnaire. All participants completed an attention network test (ANT), which measured executive control, orienting, and alerting networks.

Results The open-skill group had significantly higher executive network efficiency compared to the closed-skill $(p<0.01)$ and sedentary $(p<0.01)$ groups. The closed-skill group had significantly higher values compared to the sedentary control group $(p<0.05)$. Differences were not detected among groups for alerting and orienting networks ( $p>0.05$ ). The open-skill group had significantly higher values compared to the sedentary control group regarding proportion score of executive network $(p<0.01)$. In comparison, no significant differences were detected among groups for proportion scores of alerting and orienting networks.

Conclusion This study extends current knowledge by demonstrating that open-skill exercises selectively enhance the executive control of attentional networks in older adults. Open-skill exercises combines physical exercise and cognitive training, potentially representing a more effective exercise mode to maintain or enhance attentional function in older adults. 


\section{Exercise Mode and Attentional Networks in Older}

4 Adults: A Cross-sectional Study

Biye Wang ${ }^{1,2}$, Wei Guo ${ }^{1,2}$

${ }^{1}$ College of Physical Education, Yangzhou University, Yangzhou, Jiangsu, China

Corresponding Author:

Wei Guo

No.88 South Daxue Road, Yangzhou, Jiangsu, 225127, China

Email address: guowei_sus@outlook.com

\section{Abstract}

Background Previous studies have indicated that physical exercise enhances attentional function; however, the relationship between exercise mode and attentional networks has not been clarified for older adults ( $>60$ years old). This study aimed to investigate the relationship between attentional networks and different exercise modes in older adults.

Methods Two hundred and fifty-nine participants aged between 60 to 81 years were enrolled and classified into three groups (closed-skill group, open-skill group, or sedentary control group) using an exercise-related questionnaire. All participants completed an attention network test (ANT), which measured executive control, orienting, and alerting networks.

Results The open-skill group had significantly higher executive network efficiency compared to the closed-skill $(\mathrm{p}<0.01)$ and sedentary $(\mathrm{p}<0.01)$ groups. The closed-skill group had significantly higher values compared to the sedentary control group $(\mathrm{p}<0.05)$. Differences were not detected among groups for alerting and orienting networks $(\mathrm{p}>0.05)$. The open-skill group had significantly higher values compared to the sedentary control group regarding proportion score of executive network $(\mathrm{p}<0.01)$. In comparison, no significant differences were detected among groups for proportion scores of alerting and orienting networks.

Conclusion This study extends current knowledge by demonstrating that open-skill exercises selectively enhance the executive control of attentional networks in older adults. Open-skill exercises combines physical exercise and cognitive training, potentially representing a more effective exercise mode to maintain or enhance attentional function in older adults. 


\section{Introduction}

38 Age-related impairments in attention, memory, processing speed, and reasoning arise during

39 adulthood, and progress into the elder years (Salthouse 2016). Attention is the basic brain

40 function to ensure that we interact effectively with the environment by selectively focusing on

41 relevant information over other information (Posner \& Petersen 1990); however, this function is

42 particularly age-sensitive (Gamboz et al. 2010; Waszak et al. 2010). However, there is increasing

43 evidence that physical exercise has a positive impact on attention. For example, a recent

44 systematic review of 44 articles (with effect sizes ranging from 0.2 to 0.8 ) showed that acute

45 exercise $(n=23)$, aerobic exercise intervention $(n=9)$, and aerobic fitness $(n=12)$ might have a

46 positive effect on sustained and selective attention (Fernandes M. de Sousa et al. 2018). Another

47 recent review (Muiños \& Ballesteros 2018) also showed that older adults who participate in ball

48 sports or fast-moving sports (e.g., tennis and martial arts) exhibit better visual attention

49 compared to sedentary adults; thus, exercise mode might be related to attention function. Also, a

50 meta-analysis of randomized control trials demonstrated a significant improvement in the

51 attention and processing speed of older adults who participated in Tai Chi compared to sedentary

52 controls (Kelly et al. 2014).

53 According to attentional network theory, the human attention system is divided into three

54 networks: orienting, alerting, and executive control (Petersen \& Posner 2012; Posner \& Petersen

55 1990). Our basic attention function for selecting information from multiple sensory inputs is

56 facilitated by the orienting network, which depends on a network composed of the superior

57 parietal lobe, superior colliculus, frontal eye fields, and temporoparietal junction (Mayer et al.

58 2004). The alerting network is mediated by the parietal areas of the dorsal visual pathway and

59 the right frontal (Marzo et al. 2014), and is associated with enhancing vigilance and

60 preparedness. The executive control network is involved in monitoring and resolving conflict. It

61 is believed that anterior cingulate cortex, the dorsolateral prefrontal cortex, and portions of the

62 basal ganglia and the thalamus are associated with this network (Crottaz-Herbette \& Menon

63 2006). The attention network test (ANT) was developed to assess the attentional networks

64 independently in a single test, based on attentional network theory (Fan et al. 2002).

65 ANT has been used by previous studies to show the positive effects of martial arts training

66 (Johnstone \& Marí-Beffa 2018), table tennis training (Wang et al. 2016), chronic exercise (Pérez

67 et al. 2014), and acute bouts of aerobic exercise (Chang et al. 2015; Huertas et al. 2011) on the 
68 three components of attentional networks in young adults. A recent randomized control trial

69 showed that hatha yoga had no significant effect as an exercise intervention in older adults 70 compared to a stretching control group regarding the three attentional networks (Gothe et al.

71 2017). In contrast to open-skill exercises (e.g., table tennis and badminton), yoga is a typical

72 closed-skill exercise that is conducted in a relatively stable, predictable and self-paced

73 environment. Open-skill exercises are those in which exercisers are required to react in a

74 dynamic changing, unpredictable, and external-paced environment (McMorris 2014). Several

75 studies have demonstrated additional cognitive benefits of open-skill exercises over closed-skill

76 exercises in older adults (Dai et al. 2013; Guo et al. 2016). The additional cognitive benefits of

77 open-skill exercises are mainly attributed to environmental enrichment and increased cognitive

78 demand during physical exercise (Fabel et al. 2009). Diamond \& Ling (2016) argued that aerobic

79 exercise lacks cognitive components, and so produces little or no cognitive benefit (Diamond \&

80 Ling 2016). The authors suggested that physical training should extend beyond simple

81 movement to movement with thought (Diamond 2015). Thus, the present study aimed to

82 differentiate the relationship between exercise mode and attentional networks in older adults in 83 relation to open- and closed-skill exercise.

84 Several studies have demonstrated that advanced aging is accompanied by declines in 85 attention, with physical exercise delaying such declines. However, information on how different 86 exercise modes affects the cognitive domain in the elderly remains limited. Thus, the current

87 study aimed to extend existing knowledge by elucidating whether different exercise modes 88 produce different benefits (i.e., improvements) on certain components of attention network 89 function. Specifically, this study focused on how two exercise modes affect attention network 90 function in older adults when performing ANT. Based on existing information from the 91 published literature, we hypothesized that: (1) both open-skill and closed-skill exercisers would 92 perform better than sedentary controls in ANT; and (2) open-skill exercisers would be selectively 93 better than closed-skill exercisers with respect to executive control network function. 94 95 
96 Materials \& Methods

97 Ethical approval

98 The study was carried out ethically, and was approved by the Ethical Committee of Shanghai 99 University of Sport (No. 2017044).

100 Participants

101 Participants aged between 60 to 81 years were recruited from senior centers of various 102 communities through posted advertisements. All participants were required to satisfy the following 103 criteria: (1) right handed, (2) had normal or corrected to normal visual acuity, (3) demonstrated no 104 dementia by scoring higher than 25 in the Mini-Mental Status Examination (MMSE), (4) had a 105 normal body mass index (BMI was less than 30.0 and more than 18.5), and (5) reported to be free 106 of brain injury, psychiatric, neurological disorders, and cardiovascular disease. Fifteen applicants 107 were excluded from participation in this step. Eligible participants were categorized into three 108 groups (open-skill, closed-skill, and sedentary) according to their exercise modes, which were 109 assessed by the International Physical Activity Questionnaire (IPAQ, Chinese Version) and an 110 exercise mode questionnaire.

111 The Chinese Version of IPAQ has been proven to have good reliability and validity (Liou et 112 al. 2009), and the exercise mode questionnaire was used to obtain details of exercise, including 113 specific exercise mode, frequency per week, duration (per session), and the number of years 114 participating in certain modes of exercise. The two questionaires were adopted in previous studies 115 (Dai et al. 2013; Huang et al. 2014; Guo et al. 2016). The open-skill group satisfied the criteria of 116 participating in open-skill exercise (i.e., table tennis, badminton) at least three times per week for 11730 min each time in the previous year. The closed-skill group participated in closed-skill exercise 118 (i.e., jogging, swimming) with the same frequency. Participants who took part in both open-skill 119 and closed-skill exercises were excluded. The sedentary group reported inactivity or low activity 120 levels in IPAQ, and no regular exercise.

121 According to the results of previous meta-analyses (Northey et al. 2017; Young et al. 2015), 122 the effect size of physical exercise on cognitive function is about 0.2. A power analysis based on 123 the effect size was conducted. The result showed that a minimum of 246 subjects would be needed 124 to reach a power of 0.80 to detect the effect size of 0.2 . Significance was set to 0.05 . In total, 259 125 participants were enrolled in this study.

126 Attention network test 
The attention network test (ANT) was used to assess the function of the three attention

128

129

130

131

132

133

134

135

136

137

138

139

140

141

142

143

144

145

146

147

148

149

150

151

152

153

154

155

156

157 networks. Each trial began with a fixation cross being presented in the center of the screen. Cues were presented after a random interval of 400 to $1600 \mathrm{~ms}$, as one of the four possible conditions: no cue, center cue, double cue, or spatial cue. In the center cue condition, fixation was replaced by an asterisk. In the double cue condition, the two asterisks were respectively displayed above and below the fixation. In the spatial condition, an asterisk was displayed either above or below the fixation. These asterisks served as cues to provide temporal information about the coming target stimuli, and remained visible for $100 \mathrm{~ms}$. The spatial cue provided additional valid information about the location of target stimuli. The fixation cross was subsequently presented for $400 \mathrm{~ms}$. Then, a target stimulus was presented in a certain position according to the previous cue. In the center cue and spatial cue conditions, target stimuli were presented in the same position as a cue. In the center cue and double cue conditions, target stimuli were presented in the center of the screen. Five target stimuli were arranged as horizontal arrows or lines that were severed as flankers under three different conditions: congruent condition (the other four arrows pointed in the same direction as the central arrow), incongruent condition (the other four arrows pointed in the opposite direction to the central arrow), or neutral condition (the four lines had no directional information). Target stimuli were maintained for $1700 \mathrm{~ms}$ until the participant responded.

Participants were instructed to respond to the direction of the central arrow as fast and as accurately as possible. The participants pressed key " 1 " of a numeric keyboard with their left index fingers if the arrow pointed left, and key " 3 " with their right index fingers if the arrow pointed right. The numerical keyboard was aligned with the middle of the screen. Participants were required to concentrate on the fixation cross throughout the task.

ANT consisted of four blocks of 48 trials per person (192 in total). Within each block, all 48 trials were unique combinations of four cues condition (no cue, center cue, double cue, and spatial cue), three flanker conditions (congruent, incongruent, and neutral), two arrow directions (left or right), and two target display locations (above or below the fixation cross). ANT was presented and the data were recorded by Psychtoolbox (Fig.1).

\section{(insert Fig. 1 about here)}

\section{Procedure}

The experimenter informed the participants about the purpose of the experiment after they 
158 arrived at the laboratory. Then, the participants were instructed to sign the consent form and 159 complete the IPAQ, MMSE, and exercise mode questionnaires. Edinburgh Handedness Inventory 160 (Oldfield, 1971) and Waterloo Footedness Questionnaire Revised (WFQ-R; Elias, et al., 1998) 161 were also used to measure the laterality of the participants. Eligible participants started the ANT 162 task after reading the ANT instructions by themselves. The ANT task was performed individually 163 in a quiet and dimly lit room. All eligible participants performed a practice block consisting of 24 164 random trials. The test program counted their response accuracy (RA), and provided feedback at 165 the end of the practice block. If the RA did not reach $80 \%$, they had to perform another practice 166 block. Participants with an RA exceeding 80\% were allowed to perform the experimental blocks. 167 There were four experimental blocks for each participant. Each experimental block consisted of 16848 trials. When a block was completed, participants could take a break until they felt adequately 169 rested. The next block was initiated by pressing any key. No feedback was provided during the experimental blocks. The whole ANT task took about 17 min to complete for each participant.

\section{Design and statistical analysis}

The present study used a mixed factor design. The between-subjects variable was the exercise mode group. There were two within-subject variables: the cue type and flankers type. The dependent variables were reaction times (RTs) and accuracy rates. A group (3-open, closed, and control) $\times$ cue type (4-no, central, double, and spatial) $\times$ flanker type (3-congruent, incongruent, and neutral) mixed-design ANOVA was used to analysis the variables.

The efficiency of alerting was computed with RTs with no cue minus RTs with double cues, orienting was RTs with central cues minus RTs with spatial cues, and conflict resolution was RTs of incongruent flankers minus RTs of congruent flankers. The effect of different exercise modes on the three components of the attentional networks was evaluated using one-way ANOVA.

Receiver operating characteristic (ROC) analysis was conducted to evaluate whether the ANT 183 components could discriminate older adults that used different exercise modes, and the area under the ROC curve (AUC) was calculated. 


\section{Results}

\section{Demographic characteristics}

190 No significant differences were found in age $\left(\mathrm{F}_{(2,256)}=1.10, \mathrm{p}=0.33\right)$, education level $\left(\mathrm{F}_{(2,256)}=\right.$ 191 1.11, p = 0.33), height $\left(\mathrm{F}_{(2,256)}=1.93, \mathrm{p}=0.15\right)$, weight $\left(\mathrm{F}_{(2,256)}=1.47, \mathrm{p}=0.23\right), \operatorname{BMI}\left(\mathrm{F}_{(2,256)}\right.$ $192=0.21, \mathrm{p}=0.81)$, and $\operatorname{MMSE}\left(\mathrm{F}_{(2,256)}=1.82, \mathrm{p}=0.17\right)$. However, a significant difference was 193 observed for physical activity level $\left(\mathrm{F}_{(2,256)}=18.46, \mathrm{p}<0.01\right)$. A LSD post-hoc comparison 194 showed open- and closed- skill groups conducted a higher level of physical exercise compared to 195 the sedentary group $(\mathrm{p}<0.01)$, and that there were no significant difference between the open and 196 closed-skill groups. The main characteristics of the participants are shown in Table 1.

197

198

199

200

201

202

203

204

205

206

207

208

209

210

211

212

213

214

215

216

217

218

(insert Table 1 about here)

\section{Mean reaction times}

All incorrect trials or the trials that were three standard deviations from the individual mean were excluded from the RT analysis. A significant main effect of group $\left(\mathrm{F}_{(2,256)}=7.79, \mathrm{p}<0.01\right.$, $\left.\eta_{\mathrm{p}}{ }^{2}=0.06\right)$ was obtained. Post hoc comparison showed that open- and closed-skill groups were faster compared to the sedentary group. A significant main effect was also observed for flanker type $\left(\mathrm{F}_{(2,512)}=1285.12, \mathrm{p}<0.01, \eta_{\mathrm{p}}^{2}=0.83\right)$ and cue type $\left(\mathrm{F}_{(3,768)}=188.50, \mathrm{p}<0.01, \eta_{\mathrm{p}}{ }^{2}=\right.$ 0.42). RTs were longest under the no cue condition, and shortest under the spatial cue condition. RTs were longer under the incongruent condition compared to the congruent and neutral conditions. Significant interactions between flanker type and cue type $\left(\mathrm{F}_{(6,1536)}=18.16, \mathrm{p}<0.01\right.$, $\left.\eta_{\mathrm{p}}{ }^{2}=0.07\right)$, and group and flanker type $\left(\mathrm{F}_{(4,1024)}=18.72, \mathrm{p}<0.01, \eta_{\mathrm{p}}{ }^{2}=0.13\right)$ were obtained. The interaction analysis for flanker type and group showed that the open-skill exercise group was faster compared to the closed-skill exercise group and the sedentary group under incongruent conditions. In comparison, no differences were detected among the groups under the congruent and neutral conditions. The interaction analysis for cue and flanker type showed significant differences between the congruent and incongruent conditions, and the incongruent and neutral conditions, under all cue conditions. No significant differences between the congruent and neutral conditions were detected under any cue conditions. There was no significant interaction effect of group $\times$ cue type $\left(F_{(6,1536)}=1.23, p=0.29, \eta_{p}^{2}=0.01\right)$, or group $\times$ cue type $\times$ flanker type $\left(F_{(12,3072)}=1.01\right.$, $\left.\mathrm{p}=0.44, \eta_{\mathrm{p}}{ }^{2}=0.01\right)$. The descriptive data of the mean RTs and standard deviations of different 
219 220

221

222

223

224

225

226

227

228

229

230

231

232

233

234

235

236

237

238

239

240

241

242

243

244

245

246

247

248

249

exercise mode groups according to the cue and flanker type are shown in Table 2.

(insert Table 2 about here)

\section{Accuracy}

Significant main effects of group $\left(\mathrm{F}_{(2,256)}=4.47, \mathrm{p}=0.01, \eta_{\mathrm{p}}{ }^{2}=0.03\right)$ and flanker type $(\mathrm{F}$ $\left.(2,512)=86.09, \mathrm{p}<0.01, \eta_{\mathrm{p}}^{2}=0.25\right)$ were obtained in the accuracy analysis. There was no significant main effect of cue type $\left(\mathrm{F}_{(3,768)}=0.30, \mathrm{p}=0.82, \eta_{\mathrm{p}}{ }^{2}<0.01\right)$. There was no significant interaction between group and flanker type $\left(\mathrm{F}_{(4,1024)}=1.10, \mathrm{p}=0.36, \eta_{\mathrm{p}}{ }^{2}=0.01\right)$, flanker type and cue type $\left(\mathrm{F}_{(6,1536)}=2.03, \mathrm{p}=0.11, \eta_{\mathrm{p}}{ }^{2}=0.01\right)$, group $\times$ cue type $\left(\mathrm{F}_{(6,1536)}=2.38, \mathrm{p}=0.10\right.$, $\left.\eta_{\mathrm{p}}{ }^{2}=0.02\right)$, or group $\times$ cue type $\times$ flanker type $\left(\mathrm{F}_{(12,3072)}=1.85, \mathrm{p}=0.21, \eta_{\mathrm{p}}{ }^{2}=0.01\right)$. The descriptive data for the mean accuracy and standard deviations of the exercise mode groups according to cue and flanker type are shown in Table 3.

\section{(insert Table 3 about here)}

\section{Group differences on the efficiency of three attentional networks}

One-way ANOVA was carried out on the efficiency of each component of the attentional system (alerting, orienting, and executive networks). A significant difference among the groups was obtained for the executive network $\left(\mathrm{F}_{(2,256)}=20.78, \mathrm{p}<0.01\right)$. Post hoc analysis showed that open-skill exercisers reached a significantly higher executive network efficiency compared to closed-skill exercisers $(p<0.01)$ and sedentary controls $(p<0.01)$, and that closed-skill exercisers were significant higher than sedentary controls $(\mathrm{p}<0.01)$. No significant differences were observed for alerting $\left(\mathrm{F}_{(2,256)}=0.64, \mathrm{p}=0.53\right)$ and orienting $\left(\mathrm{F}_{(2,256)}=1.62, \mathrm{p}=0.20\right)$ networks (Fig. 2).

\section{(insert Fig. 2 about here)}

Given that a marginally significant difference occurred in the overall reaction times among the groups $\left(\mathrm{F}_{(2,256)}=7.79, \mathrm{p}<0.01\right.$, the RTs of the open-skill, closed-skill, and control groups were $618 \mathrm{~ms}, 638 \mathrm{~ms}$, and $664 \mathrm{~ms}$, respectively), the efficiency of the three attentional networks 
250

251

252

253

254

255

256

257

258

259

260

261

262

263

264

265

266

267

268

269

270

271

272

273

274

275

276

277

278

279

280

281

was divided by the overall RT of each participant. This efficiency is called the proportion scores of the attentional networks (Gamboz et al. 2010). There was a significant difference among the groups for the proportion scores in the executive network $\left(\mathrm{F}_{(2,256)}=15.99, \mathrm{p}<0.01\right)$. Post hoc analysis showed that open-skill exercisers had a significantly higher proportion score compared to sedentary controls $(\mathrm{p}<0.01)$. In comparison, no significant differences were observed for alerting $\left(\mathrm{F}_{(2,256)}=1.73, \mathrm{p}=0.18\right)$ and orienting $\left(\mathrm{F}_{(2,256)}=2.22, \mathrm{p}=0.11\right)$ networks (Fig. 3$)$.

(insert Fig. 3 about here)

\section{Receiver operating characteristic (ROC) analysis}

To evaluate whether the ANT components could discriminate older adults that used different exercise modes, receiver operating characteristic (ROC) analysis was conducted, and the area under the ROC curve (AUC) was calculated. Between the closed-skill group and the control group, the AUC value of the alerting network (AUC value $=0.46, \mathrm{p}=0.41,95 \% \mathrm{CI}, 0.38-0.55$ ), orienting network $($ AUC value $=0.48, \mathrm{p}=0.58,95 \% \mathrm{CI}, 0.39-0.56)$, and executive network (AUC value $=$ $0.58, \mathrm{p}=0.09,95 \% \mathrm{CI}, 0.49-0.66)$ was not significant. Between the open-skill group and the control group, the AUC value of the alerting network (AUC value $=0.43, \mathrm{p}=0.13,95 \% \mathrm{CI}, 0.35-$ 0.52 ) and orienting network (AUC value $=0.44, p=0.20,95 \%$ CI, 0.36-0.53) was not significant. However, the AUC value of the executive network (AUC value $=0.77, \mathrm{p}<0.01,95 \%$ CI, 0.700.84) was significant. Between the closed-skill group and the open-skill group, the AUC value of alerting network (AUC value $=0.46, \mathrm{p}=0.38,95 \% \mathrm{CI}, 0.37-0.55)$ and orienting network (AUC value $=0.47, \mathrm{p}=0.46,95 \% \mathrm{CI}, 0.38-0.55)$ was not significant. However, the AUC value of the executive network (AUC value $=0.69, \mathrm{p}<0.01,95 \% \mathrm{CI}, 0.61-0.77$ ) was significant (Fig. 4). According to Hosmer, Lemeshow, and Sturdivant (2013), AUC > 0.7 represents a good discrimination criterion (Hosmer Jr et al. 2013).

(insert Fig. 4 about here)

\section{Discussion}

The current study aimed to evaluate the relationship between different exercise modes and the attentional networks of older adults ( $>60$ years old). Two-hundred and fifty-nine older adults were allocated in the open-skill group, closed-skill group, or sedentary group based on the 
282 exercise mode questionnaire. Group differences on the executive network were found in the 283 ANOVA analysis. The interaction showed that the open-skill exercisers were faster under the 284 incongruent conditions compared to closed-skill exercisers and sedentary controls. Of note, 285 reduced executive control was accompanied by slower processing speeds; consequently, it was 286 possible to calculate the proportion scores of the attentional networks. After controlling for 287 general processing speed, open-skill exercisers exhibited better executive control compared to 288 non-exercisers. Alerting and orienting networks were similar among the groups, which was 289 consistent with a previous study showing that young adult chronic exercisers exhibit equivalent 290 levels of alerting and orienting to their sedentary controls (Pérez et al. 2014). To strengthen the 291 effect of selective enhancement, ROC analysis showed that the executive network had a larger 292 power than the alerting and orienting network at discriminating between open-skill exercisers 293 and the controls in older adults. These results are similar with a previous study showing that 294 college-level table tennis athletes exhibit selectively enhanced executive control of the

295 296 297 298 299 300 301 302 303 304 305 306 307 308 309 310 311 attentional networks (Wang et al. 2016). Thus, the selective enhancement of the executive control network of open-skill exercise occurs in both younger and older adults.

Aging is associated with the impairment of attention (Verhaeghen \& Cerella 2002). Studies that focused on the relationship between aging and the three attentional networks showed that alerting, orienting, and executive function are differentially affected by aging. In general, these studies concluded that the orienting network remains intact with aging (Fernandez-Duque \& Black 2006; Gamboz et al. 2010; Jennings et al. 2007; Williams et al. 2016). However, the results obtained for the other two networks are less consistent. For example, studies focusing on the age differences of ANT showed an age-related decline for the executive network (Gamboz et al. 2010; Jennings et al. 2007); however, when age-related slowing of reaction time was accounted for, there were no differences between younger and older individuals (FernandezDuque \& Black 2006; Williams et al. 2016). Most studies on the alerting network showed an age-related decline, with just one study reporting no age-related decline (Fernandez-Duque \& Black 2006). More specifically, one study focusing on the efficiency of attentional components in elderly people with mild neurocognitive disorders (NCD) showed that the NCD group was less efficient with respect to the executive control network, and had a slower processing speed compared to healthy elders ( $\mathrm{Lu}$ et al. 2016). 
312 Previous studies showed that attentional function is enhanced by physical exercise 313 (Hawkins et al. 1992; Sanabria et al. 2011; Tsai et al. 2016) and attention training (Tang \& 314 Posner 2009). To the best of our knowledge, our study is the first to investigate the relationship 315 between exercise mode and the attentional networks in older adults. Physical exercise was 316 divided into two modes in the present study: open-skill exercises and closed-skill exercises. 317 Compared to closed skill exercises, open-skill exercises are performed in unpredictable 318 environments that require a higher investment of cognitive effort. Open-skill exercises combine 319 physical exercise and cognitive training simultaneously (McMorris 2014). Because of their 320 321 cognitively challenging environments (Fable 2009), open-skill exercises are more effective at inducing cognitive benefits for athletes (Voss et al. 2010; Wang et al. 2013), older adults (Dai et al. 2013; Huang et al. 2014), and developmental coordination disorder (DCD) children (Tsai 2009). The results of the current study supported those focused on older adults, with open-skill exercisers exhibiting better executive function (Dai et al. 2013; Huang et al. 2014), visuospatial attention (Tsai et al. 2016), and working memory (Guo et al. 2016) compared to closed-skill exercisers.

More interestingly, no significant differences were observed between closed-skill exercisers and sedentary older adults on the proportion scores of the attentional networks in the present study. Proportion scores were used to control for the possibility that group differences in reaction time affected the efficiency of alerting, orienting, and executive control networks. The results of proportion score analyses mirrored previous studies among older adults, showing no behavioral differences between closed-skill exercisers and sedentary adults for the switch task (Dai et al. 2013) and visuospatial short-term memory (Guo et al. 2016). In comparison, significant differences were observed between open-skill exercisers and sedentary controls. This difference might be explained by the fact that these previous studies used cross-sectional designs, with a relatively small number of participants. Compared to closed-skill exercise, open-skill exercises enhanced the cognitive function of older adults in the current study.

The present study had some limitations. From the perspective of experimental design, the 339 cross-sectional design only revealed a possible relationship between the exercise mode and the attentional networks in older adults. Also, open-skill exercises require more social interaction 341 than closed-skill exercises, which might also explain the differences between the groups. Thus, 342 longitudinal studies are needed to reveal these causal relationship in the future. From the 
343 perspective of experimental implementation, the testing time was not strictly controlled. Knight 344 (2013) found that older adults performed best on the alerting network in the mornings, while the 345 other two networks were not affected by time of day (Knight \& Mather 2013). The present study 346 was performed in the morning or in the afternoon; thus, the non-significant difference in the alerting network might be masked by the timing of the tests.

\section{Conclusions}

The current study provided further evidence on the association of physical exercise with

352

353

354

better attentional networks in older adults. Compared to closed-skill exercisers and sedentary controls, open-skill exercisers exhibited a selective enhancement of the executive control network, whereas no differences were observed in the orienting and alerting networks across the three groups. The important public health implications of our results are that open-skill exercises (which combine physical exercise and cognitive training) might provide a potentially effective type of intervention for older adults to maintain or enhance attentional function.

\section{References}

Chang YK, Pesce C, Chiang YT, Kuo CY, and Fong DY. 2015. Antecedent acute cycling exercise affects attention control: an ERP study using attention network test. Front Hum Neurosci 9 Article 156.

Crottaz-Herbette S, and Menon V. 2006. Where and when the anterior cingulate cortex modulates attentional response: combined FMRI and ERP evidence. Boston: MIT Press.

Dai CT, Chang YK, Huang CJ, and Hung TM. 2013. Exercise mode and executive function in older adults: An ERP study of task-switching. Brain Cogn 83:153-162.

Diamond A. 2015. Effects of physical exercise on executive functions: going beyond simply moving to moving with thought. Annals of sports medicine and research 2:1011.

Diamond A, and Ling DS. 2016. Conclusions about interventions, programs, and approaches for improving executive functions that appear justified and those that, despite much hype, do not. Developmental cognitive neuroscience 18:34-48.

Elias LJ, Bryden MP, and Bulmanfleming MB. 1998. Footedness is a better predictor than is handedness of emotional lateralization. Neuropsychologia 36: 37-43.

Fabel K, Wolf S, Ehninger D, Babu H, Galicia P, and Kempermann G. 2009. Additive effects of physical exercise and environmental enrichment on adult hippocampal neurogenesis in mice. Front Neurosci 3 Article 2.

Fan J, Mccandliss BD, Sommer T, Raz A, and Posner MI. 2002. Testing the efficiency and independence of attentional networks. Journal of Cognitive Neuroscience 14:340-347.

Fernandes M. de Sousa A, Medeiros AR, Del Rosso S, Stults-Kolehmainen M, and Boullosa DA. 2018. The influence of exercise and physical fitness status on attention: a systematic review. International Review of Sport and Exercise Psychology:12:202-234.

Fernandez-Duque D, and Black SE. 2006. Attentional networks in normal aging and Alzheimer's disease. Neuropsychology 20:133-143.

Gamboz N, Zamarian S, and Cavallero C. 2010. Age-related differences in the attention network test (ANT). 
402

403

404

405

406

407

408

409

410

411

412

413

414

415

416

417

418

419

420

421

422

423

424

425

426

427

428

429

430

431

432

433

434

435

436

437

Experimental aging research 36:287-305.

Gothe NP, Kramer AF, and McAuley E. 2017. Hatha yoga practice improves attention and processing speed in older adults: Results from an 8-week randomized control trial. The Journal of Alternative and Complementary Medicine 23:35-40.

Guo W, Wang B, Lu Y, Zhu Q, Shi Z, and Ren J. 2016. The relationship between different exercise modes and visuospatial working memory in older adults: a cross-sectional study. PeerJ 4:e2254.

Hawkins HL, Kramer AF, and Capaldi D. 1992. Aging, exercise, and attention. Psychology and aging 7(4):643-653. Hosmer Jr DW, Lemeshow S, and Sturdivant RX. 2013. Applied logistic regression. New York: John Wiley \& Sons. Huang CJ, Lin PC, Hung CL, Chang YK, and Hung TM. 2014. Type of physical exercise and inhibitory function in older adults: An event-related potential study. Psychology of Sport \& Exercise 15:205-211.

Huertas F, Zahonero J, Sanabria D, and Lupiáñez J. 2011. Functioning of the attentional networks at rest vs. during acute bouts of aerobic exercise. Journal of Sport and Exercise Psychology 33:649-665.

Jennings JM, Dagenbach D, Engle CM, and Funke L. 2007. Age-related changes and the attention network task: An examination of alerting, orienting, and executive function. Aging, Neuropsychology, and Cognition 14:353369.

Johnstone A, and Marí-Beffa P. 2018. The effects of martial arts training on attentional networks in typical adults. Front Psychol 9 Article 80.

Kelly ME, Loughrey D, Lawlor BA, Robertson IH, Walsh C, and Brennan S. 2014. The impact of exercise on the cognitive functioning of healthy older adults: A systematic review and meta-analysis. Ageing Research Reviews 16:12-31.

Knight M, and Mather M. 2013. Look out-it's your off-peak time of day! Time of day matters more for alerting than for orienting or executive attention. Experimental aging research 39:305-321.

Liou YM, Jwo JC, Yao KG, Chiang LC, and Huang LH. 2009. Selection of Appropriate Chinese Terms to Represent Intensity and Types of Physical Activity Terms for Use in the Taiwan Version of IPAQ. The journal of nursing research 16:252-263.

Lu H, Chan SS, Fung AW, and Lam LC. 2016. Efficiency of attentional components in elderly with mild neurocognitive disorders shown by the attention network test. Dementia and geriatric cognitive disorders 41:93-98.

Marzo A, Totah NK, Neves RM, Logothetis NK, and Eschenko O. 2014. Unilateral electrical stimulation of rat locus coeruleus elicits bilateral response of norepinephrine neurons and sustained activation of medial prefrontal cortex. Journal of Neurophysiology 111:2570-2588.

Mayer AR, Dorflinger JM, Rao SM, and Seidenberg M. 2004. Neural networks underlying endogenous and exogenous visual-spatial orienting. Neuroimage 23:534-541.

McMorris T. 2014. Acquisition and performance of sports skills. New York: John Wiley \& Sons.

Muiños M, and Ballesteros S. 2018. Does physical exercise improve perceptual skills and visuospatial attention in older adults? A review. European Review of Aging and Physical Activity 15 Article 2.

Northey JM, Cherbuin N, Pumpa KL, Smee DJ , and Rattray B. 2017. Exercise interventions for cognitive function in adults older than 50: a systematic review with meta-analysis. British Journal of Sports Medicine, bjsports2016-096587.

Oldfield RC. 1971. The assessment and analysis of handedness: The Edinburgh inventory. Neuropsychologia 9: 97113.

Pérez L, Padilla C, Parmentier FB, and Andrés P. 2014. The effects of chronic exercise on attentional networks. PLoS One 9:e101478.

Petersen SE, and Posner MI. 2012. The attention system of the human brain: 20 years after. Annual review of neuroscience 35:73-89.

Posner MI, and Petersen SE. 1990. The attention system of the human brain. Annual review of neuroscience 13:2542.

Salthouse TA. 2016. Continuity of cognitive change across adulthood. Psychonomic bulletin \& review 23:932-939.

Sanabria D, Morales E, Luque A, Gálvez G, Huertas F, and Lupiañez J. 2011. Effects of acute aerobic exercise on exogenous spatial attention. Psychology of Sport and Exercise 12:570-574.

Tang Y-Y, and Posner MI. 2009. Attention training and attention state training. Trends in Cognitive Sciences 13:222227.

Tsai CL, Wang CH, Pan CY, Chen FC, Huang SY, and Tseng YT. 2016. The effects of different exercise types on 
438

439

440

441

442

443

444

445

446

447

448

449

450

451

452

453

454

455

456

visuospatial attention in the elderly. Psychology of Sport and Exercise 26:130-138.

Tsai CL. 2009. The effectiveness of exercise intervention on inhibitory control in children with developmental

coordination disorder: Using a visuospatial attention paradigm as a model. Res Dev Disabil 30:1268-1280.

Verhaeghen P, and Cerella J. 2002. Aging, executive control, and attention: a review of meta-analyses.

Neuroscience \& Biobehavioral Reviews 26:849-857.

Voss MW, Kramer AF, Basak C, Prakash RS, and Roberts B. 2010. Are expert athletes 'expert' in the cognitive laboratory? A meta-analytic review of cognition and sport expertise. Applied Cognitive Psychology 24:812826.

Wang B, Guo W, and Zhou C. 2016. Selective enhancement of attentional networks in college table tennis athletes: a preliminary investigation. PeerJ 4:e2762.

Wang CH, Chang CC, Liang YM, Shih CM, Chiu WS, Tseng P, Hung DL, Tzeng OJ, Muggleton NG, and Juan CH. 2013. Open vs. closed skill sports and the modulation of inhibitory control. PLoS One 8:e55773.

Waszak F, Li S-C, and Hommel B. 2010. The development of attentional networks: Cross-sectional findings from a life span sample. Developmental psychology 46:337-349.

Williams RS, Biel AL, Wegier P, Lapp LK, Dyson BJ, and Spaniol J. 2016. Age differences in the Attention Network Test: Evidence from behavior and event-related potentials. Brain and cognition 102:65-79.

Young J, Angevaren M, Rusted J, and Tabet N. 2015. Aerobic exercise to improve cognitive function in older people without known cognitive impairment. Cochrane database of systematic reviews (Online) 4(4): CD005381. 


\section{Table $\mathbf{1}$ (on next page)}

Main characteristics of the participants in thethree groups

Note. MMSE = Mini Mental State Exam, IPAQ= International Physical Activity Questionnaire, METs= metabolic equivalents. 
Table 1 Main characteristics of the participants in the three groups

\begin{tabular}{cccc}
\hline & $\begin{array}{c}\text { Sedentary } \\
(\mathrm{n}=87)\end{array}$ & $\begin{array}{c}\text { Closed-skill } \\
(\mathrm{n}=87)\end{array}$ & $\begin{array}{c}\text { Open-skill } \\
(\mathrm{n}=85)\end{array}$ \\
\hline Male & 41 & 38 & 40 \\
Age (years) & $65.9 \pm 6.3$ & $65.5 \pm 5.8$ & $66.8 \pm 5.5$ \\
Education (years) & $11.4 \pm 2.3$ & $10.9 \pm 2.3$ & $11.3 \pm 2.6$ \\
Height (cm) & $161.3 \pm 5.8$ & $161.8 \pm 5.5$ & $163.0 \pm 7.1$ \\
Weight (kg) & $62.3 \pm 6.0$ & $62.1 \pm 5.9$ & $63.8 \pm 9.4$ \\
BMI (kg/m $\left.{ }^{2}\right)$ & $24.0 \pm 2.3$ & $23.8 \pm 2.2$ & $24.0 \pm 2.9$ \\
MMES & $27.9 \pm 1.4$ & $28.2 \pm 1.6$ & $28.3 \pm 1.2$ \\
IPAQ(METs/week) & $4458.8 \pm 2770.8$ & $6683.9 \pm 3031.9$ & $6737.4 \pm 2631.0$ \\
\hline
\end{tabular}

2 Note. $\mathrm{MMSE}=$ Mini Mental State Exam, IPAQ= International Physical Activity Questionnaire, METs= 3 metabolic equivalents.

4 


\section{Table 2 (on next page)}

Mean RTs (ms) and standard deviations of the three exercisemode groups according to cue and flanker type 
1 Table 2 Mean RTs (ms) and standard deviations of the three exercise mode groups according to cue and 2 flanker type

\begin{tabular}{cccccc}
\hline Group & Flanker type & \multicolumn{4}{c}{ Cue type } \\
\hline \multirow{2}{*}{ Sedentary } & No cue & Center cue & Double cue & Spatial cue \\
\hline & Incongruent & $728.84 \pm 106.75$ & $730.01 \pm 99.70$ & $721.36 \pm 97.90$ & $701.28 \pm 105.1$ \\
& & & & & 1 \\
\hline \multirow{3}{*}{ Closed-skill } & Neutral & $646.17 \pm 99.03$ & $625.88 \pm 81.35$ & $630.36 \pm 93.30$ & $622.26 \pm 91.99$ \\
& Congruent & $640.06 \pm 68.25$ & $614.99 \pm 75.47$ & $617.30 \pm 73.66$ & $605.30 \pm 71.25$ \\
& Neutral & $625.32 \pm 64.10$ & $602.78 \pm 63.19$ & $605.63 \pm 66.80$ & $590.41 \pm 57.21$ \\
\hline & Congruent & $622.63 \pm 70.35$ & $599.89 \pm 73.85$ & $596.86 \pm 76.51$ & $593.05 \pm 74.06$ \\
& Incongruent & $665.28 \pm 83.70$ & $666.93 \pm 73.94$ & $654.36 \pm 79.93$ & $633.25 \pm 77.97$ \\
& Neutral & $615.44 \pm 71.65$ & $599.82 \pm 61.84$ & $590.92 \pm 70.15$ & $580.25 \pm 70.97$ \\
\hline
\end{tabular}

3

4 


\section{Table 3 (on next page)}

Mean accuracy (\%) and standard deviations of the exercise mode groups according to cue and flanker type 
1 Table 3 Mean accuracy (\%) and standard deviations of the exercise mode groups according to cue and flanker 2 type

\begin{tabular}{cccccc}
\hline \multirow{2}{*}{ Froup } & Flanker & \multicolumn{4}{c}{ Cue type } \\
\hline \multirow{2}{*}{ Sedentary } & No cue & Center cue & Double cue & spatial cue \\
& Congruent & $97.41 \pm 4.63$ & $97.99 \pm 5.99$ & $96.26 \pm 6.06$ & $98.49 \pm 3.56$ \\
& Neutral & $97.77 \pm 4.95$ & $97.49 \pm 4.81$ & $97.99 \pm 5.00$ & $96.98 \pm 4.66$ \\
\hline \multirow{2}{*}{ Closed-skill } & Incongruent & $95.69 \pm 7.88$ & $94.25 \pm 7.99$ & $95.40 \pm 6.57$ & $94.25 \pm 5.74$ \\
& Nengruent & $96.77 \pm 4.75$ & $98.78 \pm 3.28$ & $97.99 \pm 3.63$ & $97.27 \pm 4.74$ \\
& Congruent & $98.38 \pm 3.22$ & $97.94 \pm 3.25$ & $98.46 \pm 3.18$ & $98.46 \pm 3.97$ \\
& Incongruent & $95.88 \pm 4.48$ & $96.91 \pm 5.25$ & $95.44 \pm 6.35$ & $96.91 \pm 4.59$ \\
& Neutral & $97.94 \pm 2.95$ & $96.99 \pm 4.27$ & $99.26 \pm 2.24$ & $98.46 \pm 3.60$ \\
\hline \multirow{2}{*}{ Open-skill } & Nent & $97.41 \pm 5.09$ & $97.92 \pm 3.77$ & $97.77 \pm 4.15$ & $98.20 \pm 2.84$ \\
\hline
\end{tabular}

3 
Figure 1

Stimuliand experimental paradigm of the Attention Network Test (ANT)

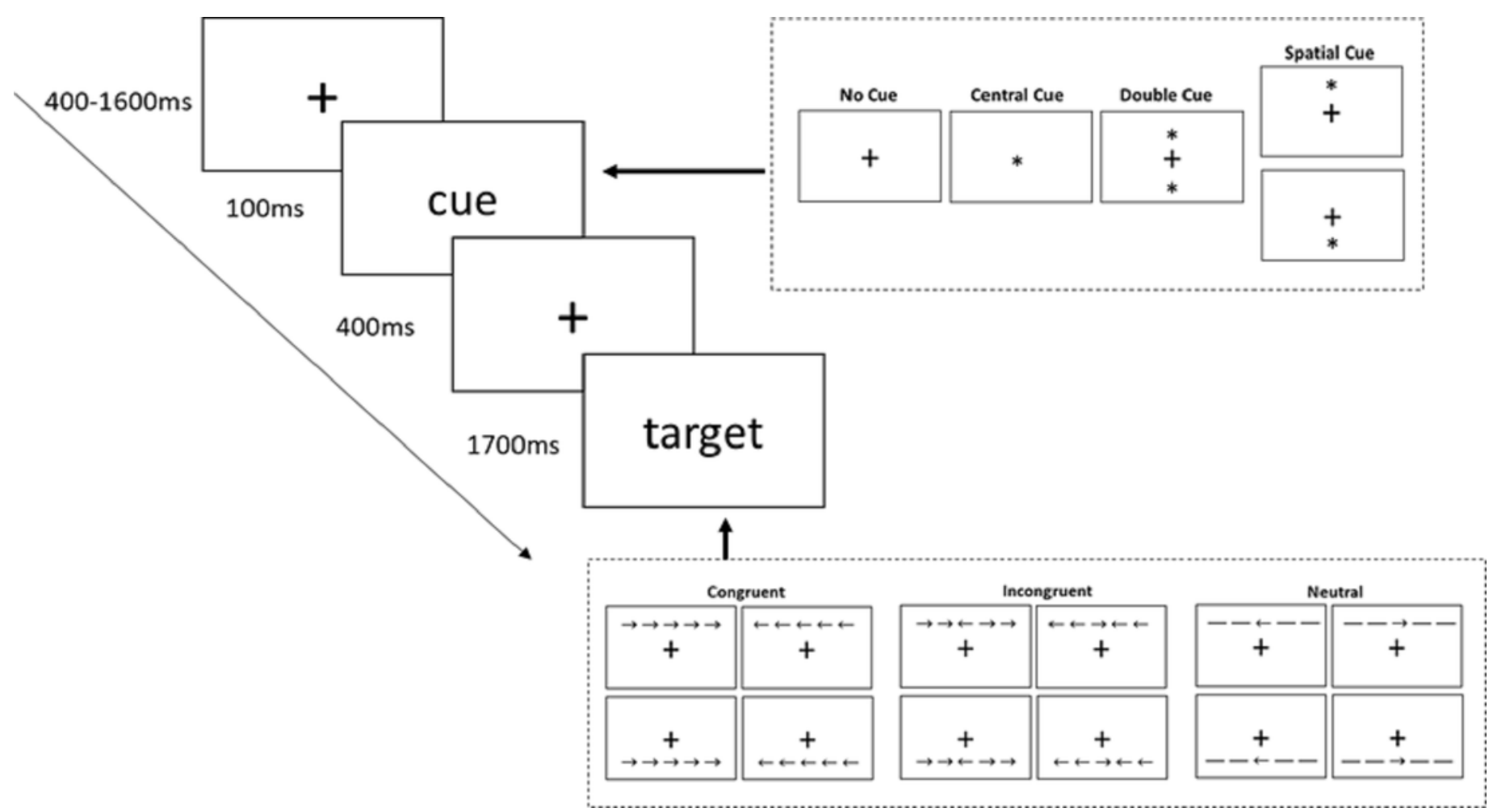


Figure 2

Efficiencyof the three attentional networks in older adults with different exercise modes

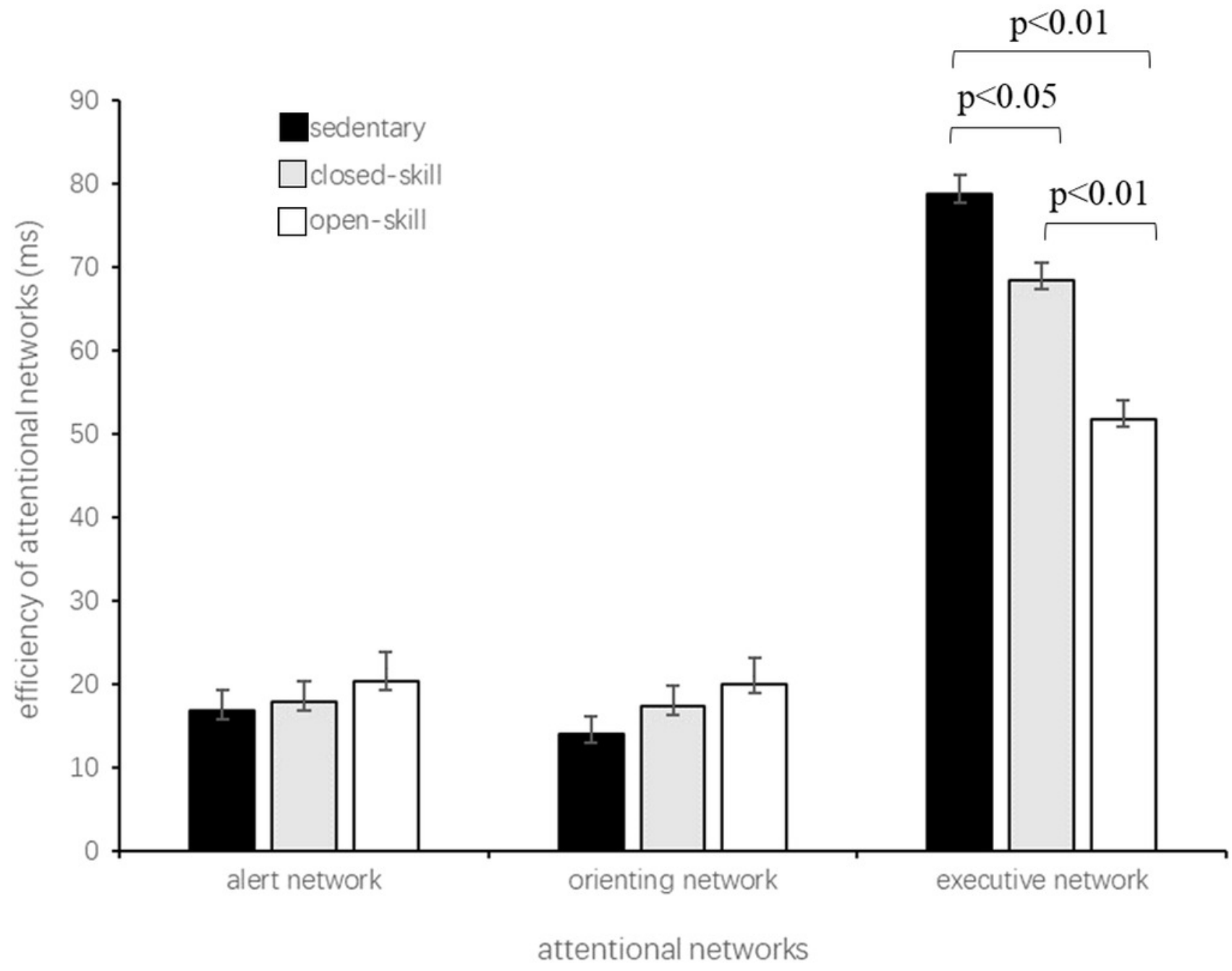


Figure 3

Proportionscores of the three attentional networks in older adults with different exercise modes

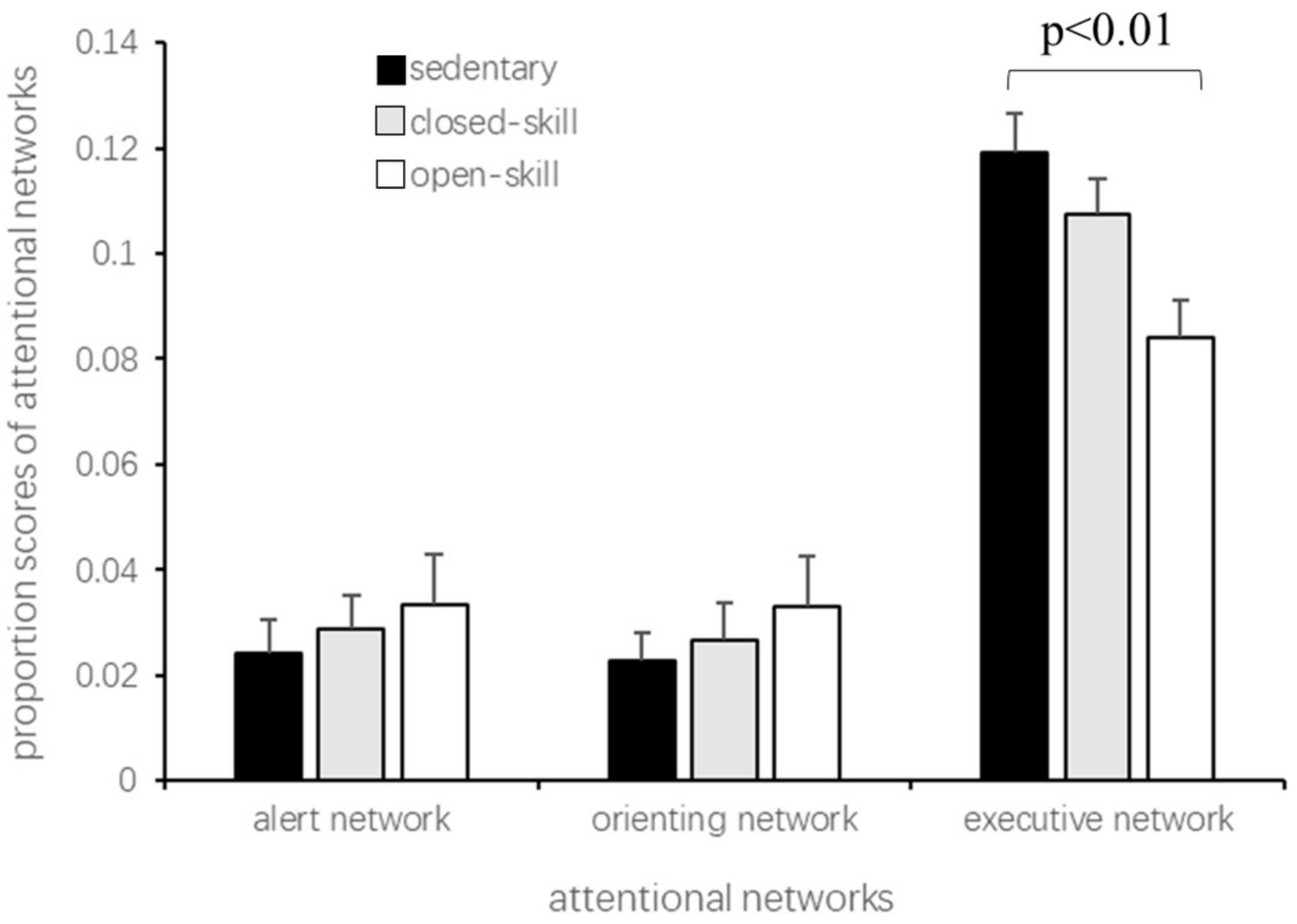


Figure 4

ROC curves for the three attentional networks

(a) the closed-skill group and the control group; (b) the open-skill group and the control group; (c) the closed-skill group and the open-skill group.
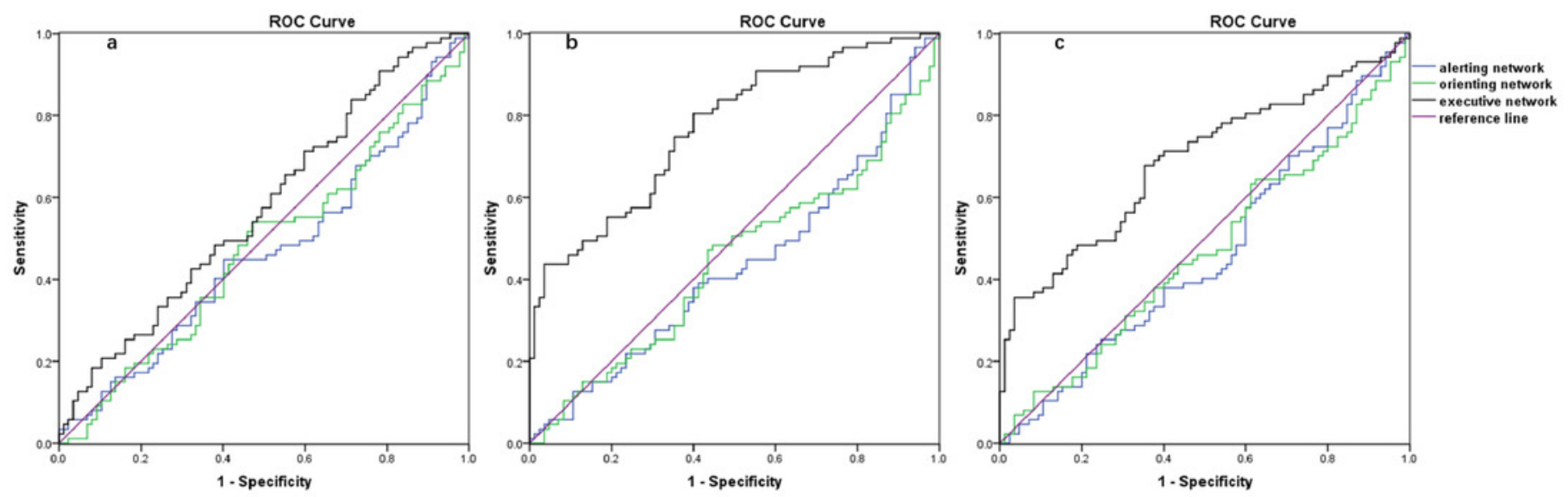